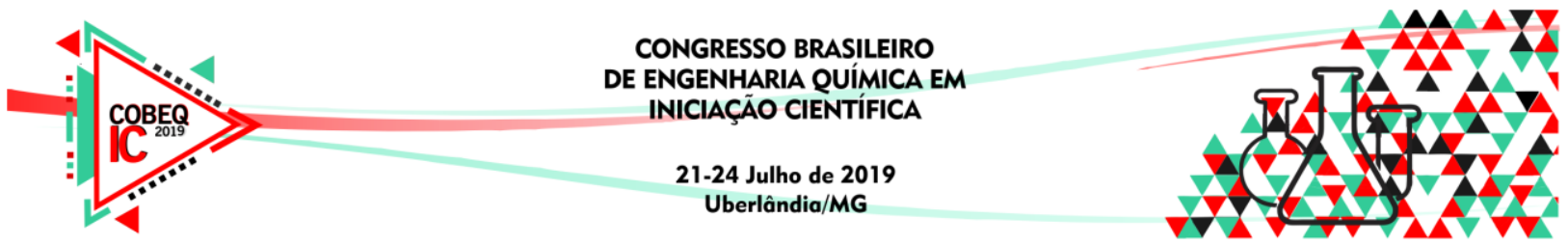

\title{
ANÁLISE DA JANELA DE VIALIBILIDADE ECONÔMICA A PARTIR DE SIMULAÇÕES EM DIFERENTES SISTEMAS DA PRODUÇÃO DE ÁCIDO CÍTRICO EM AnaBioPlus
}

\author{
L. M. GIANESINI ${ }^{1}$; A. C. O. MAFRA ${ }^{1}$ \\ ${ }^{1}$ Universidade Federal de Mato Grosso, Faculdade de Engenharia de Várzea Grande \\ E-mail para contato: ligianesini@ hotmail.com
}

\begin{abstract}
RESUMO - O objetivo deste estudo é analisar a janela de viabilidade econômica do mesmo desenho industrial, produção de ácido cítrico via fermentativa com uso da levedura doméstica Yaworria lipolytica $57 \mathrm{em}$ frutose, sob diferentes configurações do sistema. Essa pesquisa se deu através da literatura de inúmeros artigos relacionados ao tema e com auxílio do software AnaBioPlus. Após a simulação de três diferentes políticas de processo e análise das métricas de processo, observou-se que apenas uma se encaixava como caminho viável economicamente. Assim, pode-se concluir que a janela de viabilidade econômica é uma ótima ferramenta de análise para avaliações financeiras do processo industrial que se deseja estudar, no caso em questão, apenas o sistema em batelada alimentada pode ser considerado como viável econômica e industrialmente.
\end{abstract}

\section{INTRODUÇÃO}

O ácido cítrico (ácido 2-hidroxi-propano-1,2,3-tricarboxílico), dado pela fórmula química $\mathrm{C}_{6} \mathrm{H}_{7} \mathrm{O}_{8}$. É um ácido orgânico fraco de massa molecular $192,123 \mathrm{~g} / \mathrm{mol}$, que contém três grupos funcionais carboxílicos com três valores diferentes de pKa $(3.15,4.77$ e 6.4). É um produto metabólico primário formado no ciclo do ácido tricarboxílico (ou Krebs) e é encontrado em pequenas quantidades em praticamente todas as plantas, principalmente nas frutas cítricas (Max et al., 2010). Devido as suas propriedades de ser acidulante, palatabilidade, atoxicidade, facilidade de assimilação pelo organismo humano, tamponamento e sequestramento de íons, o ácido cítrico apresenta uma série de aplicações na indústria (Berovic e Legisa, 2007).

O produto é vendido em cerca de $70 \%$ de 1,5 milhão de toneladas por ano para a indústria alimentícia como acidificante ou antioxidante para preservar ou melhorar os sabores e aromas de sucos de frutas e sorvetes. $20 \%$ é utilizado na indústria farmacêutica como antioxidante para preservar vitaminas, efervescente, corretor de $\mathrm{pH}$ e conservante de sangue. Na indústria química, são usados os $10 \%$ restantes, como agente espumante para o amaciamento e tratamento de têxteis (Max et al., 2010).

Apesar de existirem métodos clássicos de produção do ácido, tendo base os estudos de Scheele (Wehmer, 1893) via isolamento dNa este de frutas cítricas e de Grimoux e Adam (1880) através da síntese química, nenhuma atingiu o status comercial competitivo com os processos de fermentação (Berovic e Legisa, 2007). E em escala industrial, o microorganismo que apresenta melhor produtividade para o processo fermentativo é o fungo 


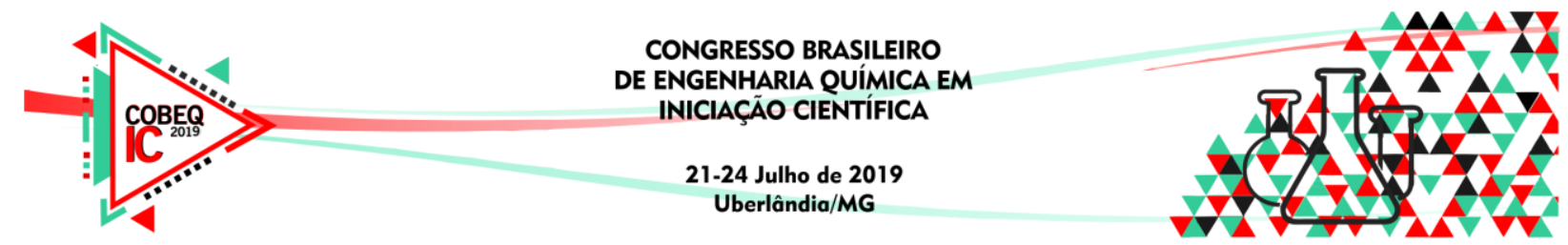

Aspergillus niger, porém durante os últimos quarenta anos o interesse dos pesquisadores tem sido atraído para produção do ácido com o uso de leveduras (Morgunov et al., 2018). Já que estas possuem potenciais vantagens em relação aos fungos: maior tolerância a altas concentrações iniciais de substrato, maior taxa de conversão, maior produtividade e permitem maior controle do processo por serem de natureza unicelular (Mattey, 1992).

Para o Engenheiro Químico, o processo industrial citado anteriormente, produção de ácido cítrico via fermentativa, deve ser visto a partir de um olhar crítico com relação a seus fins comerciais, assim, devem ser feitas avaliações econômicas com objetivo de confirmar ou não a viabilidade de produção do produto. Para tanto, o método escolhido para analisar tal parâmetro foi o da janela de viabilidade econômica (Tufvesson et al., 2013; Lima-Ramos et al., 2014), o qual faz o uso de métricas de processo para estimar os esforços de desenvolvimento necessários e a contribuição de cada passo para a viabilidade do processo. Com o objetivo de avaliar diferentes políticas de processo para encontrar o melhor caminho para a produção do ácido em estudo foi o usado o software AnaBioPlus (Oliveira et al., 2017) através da extensão SimulaFerm 1.0, este realiza simulações de biorreatores, obtendo curvas para o crescimento da célula, formação de produto e limitando o consumo de substrato.

Portanto, percebe-se a necessidade de pesquisas para a produção de ácido cítrico via fermentativa com uso de leveduras devido aos seus maiores benefícios. Assim, o presente trabalho visa analisar a janela de viabilidade econômica do processo de produção do ácido em questão em diferentes configurações, porém sempre para a mesma levedura doméstica $Y$. lipolytica 57 em frutose.

\section{METODOLOGIA}

\subsection{Métodos de abordagem e parâmetros cinéticos}

O crescimento de um micro-organismo, segundo Yalcin et al. (2009), segue a Equação 1 e para a velocidade de crescimento das células é influenciada pelas concentrações de substrato e da própria célula, apresenta tal relação em função da velocidade específica e pode ser representada por funções matemáticas hiperbólicas, a qual neste estudo é dada pela Equação 2. A taxa de crescimento específica pode ser inibida por constituintes do meio, tais como substrato ou produto; um exemplo é dado pela Eq. 2, de Andrews (Andrews, 1968), a qual propõe inibição pelo substrato, assim como também é exposto por Bailey e Ollis (1986).

Frutose + Y.lipolytica $57 \rightarrow$ Ácido citríco + Y. lipolytica 57

$$
\mu=\mu_{m a x x} \frac{C_{S}}{K_{S}+C_{S}+\frac{C_{S}^{2}}{K_{I S}}}
$$




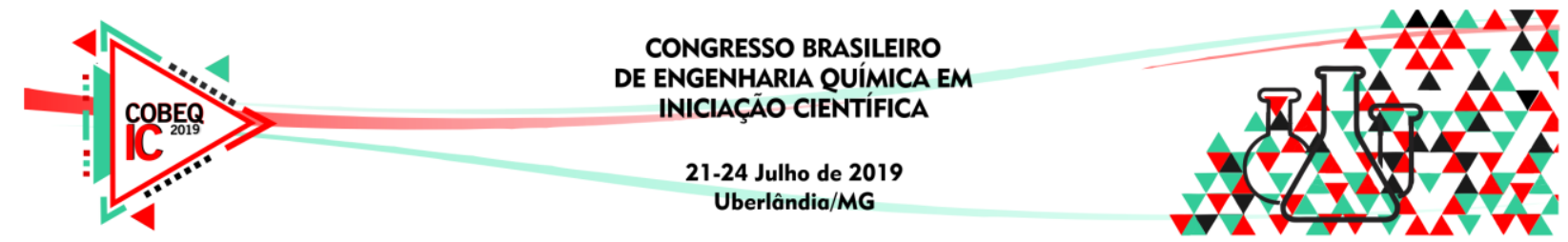

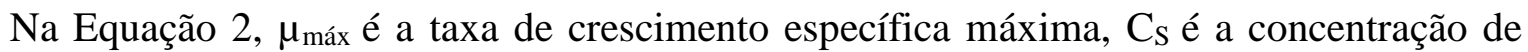
substrato inicial, $\mathrm{K}_{\mathrm{S}}$ é a constante de saturação do modelo de Monod e $\mathrm{K}_{\mathrm{IS}}$ é a constante de dissociação do substrato.

Foram usados os parâmetros cinéticos do artigo A comparative study on citric acid production kinects of two Yarrowia lipolytica strains in two different media dos autores S. Karau Yalcin, M. T. Bozdemir e Z. Y. Ozbas, os quais estão apresentados na Tabela 1. Os parâmetros cinéticos escolhidos foram do processo que apresentou maior concentração de ácido cítrico, ou seja, do cenário fermentativo em batelada com frutose como substrato e a levedura doméstica Yaworria lipolytica 57 (Yalcin et al., 2009).

Tabela 1 - Parâmetros cinéticos

\begin{tabular}{|l|c|}
\hline Concentração inicial de células $\left(\boldsymbol{C} \boldsymbol{x}_{0}\right)$ & $1 \mathrm{~g} / \mathrm{L}$ \\
\hline Concentração inicial de substrato $\left(\boldsymbol{C} \boldsymbol{s}_{0}\right)$ & $200 \mathrm{~g} / \mathrm{L}$ \\
\hline Tempo de cultivo $(\boldsymbol{t})$ & $300 \mathrm{horas}$ \\
\hline Coeficiente de rendimento de substrato em células $\left(\boldsymbol{Y}_{\boldsymbol{x} / \boldsymbol{s}}\right)$ & 0,089 \\
\hline Coeficiente de rendimento de substrato em produto $\left(\boldsymbol{Y}_{\boldsymbol{p} / \boldsymbol{s}}\right)$ & 0,3754 \\
\hline Velocidade específica máxima de crescimento celular $\left(\boldsymbol{\mu}_{\max }\right)$ & $0,069(1 / \mathrm{h})$ \\
\hline Constante de Andrews $\left(\boldsymbol{K}_{\boldsymbol{s}}\right)$ & $69,54 \mathrm{~g} / \mathrm{L}$ \\
\hline Constante de degradação do produto $\left(\boldsymbol{K}_{I S}\right)$ & $120,03 \mathrm{~g} / \mathrm{L}$ \\
\hline
\end{tabular}

\subsection{Simulação em AnaBioPlus}

Utilizando com base os dados experimentais do artigo dos autores Yalcin, Bozdemir e Ozbas (2009), conforme a Tabela 1, foi realizada a simulação no software AnaBioPlus através da SimulaFerm 1.0.

\subsection{Análise da Janela de Viabilidade Econômica}

A análise da janela de viabilidade econômica foi realizada baseando-se na Tabela 2.

Tabela 2 - Valores limitantes para as métricas dos processos para janela de viabilidade econômica (adaptada de Lima-Ramos et al., 2014)

\begin{tabular}{|c|c|c|c|}
\hline Métrica do processo & $\begin{array}{c}\text { Rendimento da } \\
\text { levedura } \\
\left(\mathrm{g}_{\text {produto }} / \mathrm{g}_{\text {biocatalisador }}\right)\end{array}$ & $\begin{array}{c}\text { Concentração de } \\
\text { produto } \\
\left(\mathrm{g}_{\text {produto }} / \mathrm{L}_{\text {reator }}\right)\end{array}$ & $\begin{array}{c}\text { Produtividade } \\
\left(\mathrm{g}_{\text {produto }} / \mathrm{L}_{\text {reator }} \mathrm{h}\right)\end{array}$ \\
\hline $\begin{array}{c}\text { Produtos químicos à } \\
\text { granel }\end{array}$ & $10^{3}-10^{5}$ & $>300$ & $>10$ \\
\hline
\end{tabular}

Assim, foi realizada uma análise comparativa tendo em vista a janela de viabilidade econômica para o sistema em regime batelada do artigo base. Considerando que o processo possa não se encaixar como viável economicamente, foram propostas duas possíveis soluções, diferentes parâmetros para regime batelada e sistema em batelada alimentada, em que apenas uma conseguiu atingir o objetivo. 


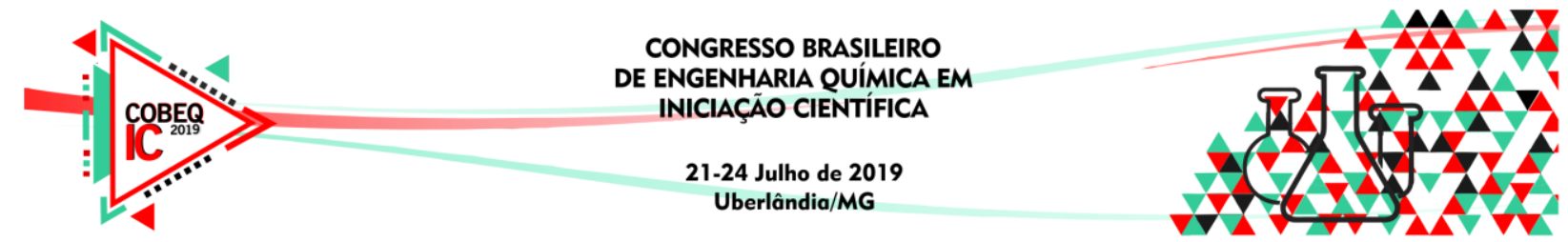

\section{RESULTADOS E DISCUSSÃO}

Realizou-se com sucesso a simulação de um processo em regime de batelada no software AnaBioPlus, usando os parâmetros de entrada descritos na Tabela 1. Com base nos dados das Tabelas 1 e 2, obtém-se a Tabela 3, a qual apresenta a janela de viabilidade econômica do primeiro processo simulado.

Tabela 3 - Dados obtidos da fermentação original do sistema em batelada para janela de viabilidade econômica

\begin{tabular}{|c|c|c|}
\hline $\begin{array}{c}\text { Rendimento do } \\
\text { biocatalisador } \\
\left(\mathrm{g}_{\text {produto }} / \mathrm{g}_{\text {biocatalisador }}\right)\end{array}$ & $\begin{array}{c}\text { Concentração de } \\
\text { produto } \\
\left(\mathrm{g}_{\text {produto }} / \mathrm{L}_{\text {reator }}\right)\end{array}$ & $\begin{array}{c}\text { Produtividade } \\
\left(\mathrm{g}_{\text {produto }} / \mathrm{L}_{\text {reator }} \mathrm{h}\right)\end{array}$ \\
\hline 75,32 & 75,32 & 0,6 \\
\hline
\end{tabular}

Pode-se concluir, então, que para este caso, o processo não é viável industrialmente. Para contornar tal problema, foi proposto a simulação do mesmo desenho industrial, porém aumentando as concentrações iniciais de substrato e de células para 1000 e $225 \mathrm{~g} / \mathrm{L}$, respectivamente, e os resultados estão dispostos na Tabela 4.

Tabela 4 - Dados obtidos da fermentação em batelada adaptada com aumento nas concentrações iniciais de substrato e células para janela de viabilidade econômica

\begin{tabular}{|c|c|c|}
\hline $\begin{array}{c}\text { Rendimento do } \\
\text { biocatalisador } \\
\left(\mathrm{g}_{\text {produto }} / \mathrm{g}_{\text {biocatalisador }}\right)\end{array}$ & $\begin{array}{c}\text { Concentração de } \\
\text { produto } \\
\left(\mathrm{g}_{\text {produto }} / \mathrm{L}_{\text {reator }}\right)\end{array}$ & $\begin{array}{c}\text { Produtividade } \\
\left(\mathrm{g}_{\text {produto }} / \mathrm{L}_{\text {reator }} \cdot \mathrm{h}\right)\end{array}$ \\
\hline 2,16 & 486,32 & 10,4 \\
\hline
\end{tabular}

Analisando as Tabelas 2 e 4, pode-se observar que uma das métricas está fora do esperado, o que significa que o processo ainda não pode ser considerado como produzível em escala industrial. Sendo assim, foi sugerido outra mudança nos parâmetros, a qual consistia em mudar o sistema para batelada alimentada e aumentar a concentração de substrato inicial para $1000 \mathrm{~g} / \mathrm{L}$, tempo de batelada de 20 horas, vazão de alimentação de $10 \mathrm{~L} / \mathrm{h}$, volume do reator de $10 \mathrm{~L}$, tempo total de fermentação de 2000 horas, concentração de substrato e de célula de entrada no tanque de 150 e $1 \mathrm{~g} / \mathrm{L}$, respectivamente; os resultados foram anotados na Tabela 5.

Tabela 5 - Dados obtidos da fermentação adaptada para sistema em batelada alimentada e mudança nos valores dos parâmetros cinéticos para janela de viabilidade econômica

\begin{tabular}{|c|c|c|}
\hline $\begin{array}{c}\text { Rendimento do } \\
\text { biocatalisador } \\
\left(\mathrm{g}_{\text {produto }} / \mathrm{g}_{\text {biocatalisador }}\right)\end{array}$ & $\begin{array}{c}\text { Concentração de } \\
\text { produto } \\
\left(\mathrm{g}_{\text {produto }} / \mathrm{L}_{\text {reator }}\right)\end{array}$ & $\begin{array}{c}\text { Produtividade } \\
\left(\mathrm{g}_{\text {produto }} / \mathrm{L}_{\text {reator. }} \mathrm{h}\right)\end{array}$ \\
\hline 1680 & 1680 & 40,87 \\
\hline
\end{tabular}




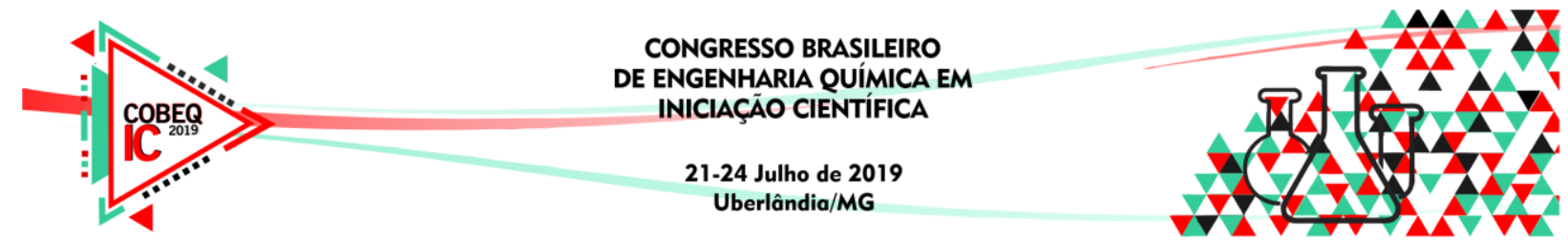

Mediante as Tabelas 2 e 5, foi possível encontrar um caminho fermentativo que se encaixasse nas métricas da janela de viabilidade econômica, ou seja, um processo viável economicamente para produção industrial. Dessa forma, através dos resultados das três simulações realizadas foi desenvolvida a Figura 1; esta conta com a janela de viabilidade econômica usando valores limites para a métrica de processo relativa à Tabela 2 .

Figura 1 - Janela de viabilidade econômica para produtos químicos a granel

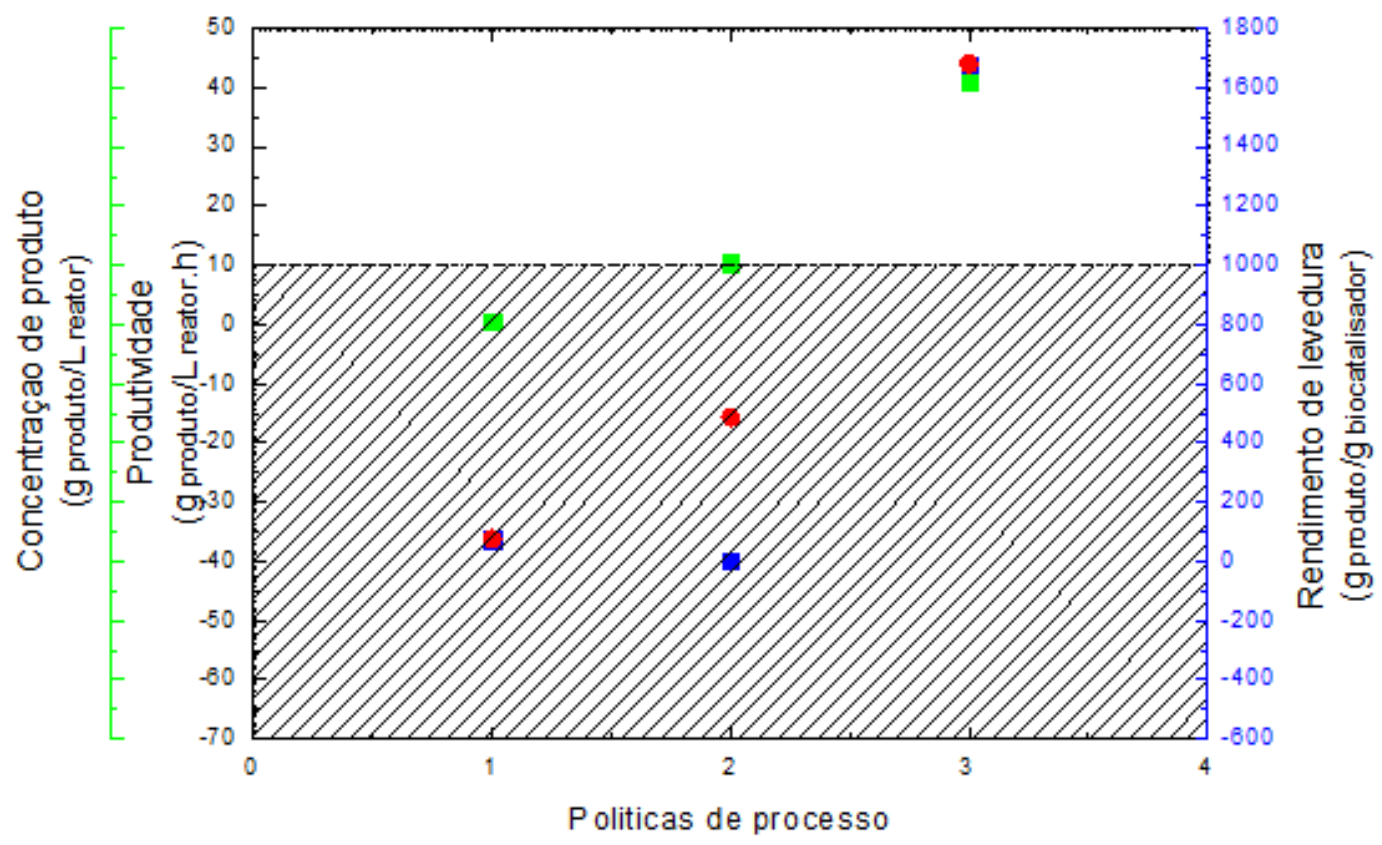

\section{CONCLUSÃO}

Nota-se, então, que o método de abordagem utilizado foi satisfatório, visto que os parâmetros cinéticos coletados do artigo dos autores Yalcin, Bozdemir e Ozbas (2009) foram simulados com robustez, através do software AnaBioPlus (Oliveira et al., 2017), uma vez que os perfis de consumo de substrato e formação de produto gerados foram similares.

A partir das simulações foi possível realizar a avaliação econômica do processo industrial para distintas políticas de processo, duas com diferentes parâmetros para regime em batelada e outra para batelada alimentada, concluindo-se que apenas o último se encaixa como processo viável em produção de escala industrial ao comparar com a janela de viabilidade econômica.

\section{AGREDECIMENTOS}

Agradeço à Universidade Federal de Mato Grosso e à Professora $\operatorname{Dr}^{\mathrm{a}}$. Agnes Cristina Oliveira Mafra pela orientação e disponibilidade durante todo o processo de produção do artigo. 


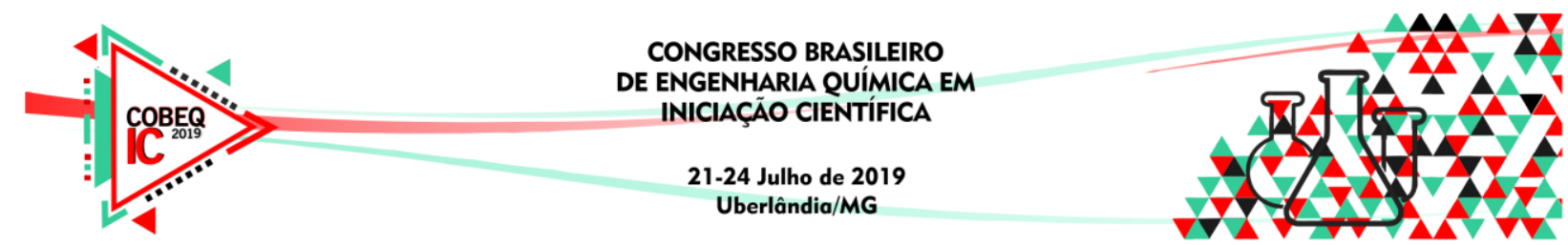

\section{REFERÊNCIAS BIBLIOGRÁFICAS}

ANDREWS, J. F. A mathematical model for the continuous culture of microorganisms utilizing inhibitory substrates. Biotechnology and Bioengineering, v. 10, p. 707-723, 1968.

BAILEY, J. E.; OLLIS, D. F. Biochemical engineering fundamentals. McGrawHill, New York, 1986.

BEROVIC, M.; LEGISA, M. Citric acid production. Biotechnology anual review, v. 13, p. 303-343, 2007.

GRIMOUX, E.; ADAM, P. Synthese del'acide citrique. CR Acad Sci Paris, v. 90, p. 1252-1256, 1880.

LIMA-RAMOS, J.; TUFVESSON, P.; WOODLEY, J. M. Application of environmental and economic metrics to guide the development of biocatalytic processes. Green Process Synth, v. 3, p. 195-213, 2014.

MATTEY, M. The production of organics acids. Critica Reviews in Biotechnology, v. 12, p. 87-132, 1992.

MAX, B.; SALGADO, J. M.; RODRÍGUEZ, N.; CORTÉS, S.; CONVERTI, A.; DOMÍNGUEZ, J. M. Biotechnological production of citric acid. Brazilian Journal of Microbiology, v. 41, p. 862-875, 2010.

MURGUNOV, I. G.; KAMZOLOVA, S. V.; LUNINA J. N. Citric acid production by Yaworria lipolytica yeast on different renewable raw materials. Fermentation, v. 4, p. 36, 2018.

OLIVEIRA, C. M.; JESUS, C. D. F.; CENEVIVA, L.V. S.; SILVA, F. H.; CRUZ, A. J. G.; COSTA, C. B. B.; BADINO, A. C. AnaBioPlus: a new package for parameter estimation and simulation of bioprocesses. Brazilian Journal of Chemical Engineering, v. 34, p. 1065-1082, 2017.

TUFVESSON, P.; LIMA-RAMOS, J.; HAQUE, N. A.; GERNAEY, K. V.; WOODLEY, J. M. Advances in the process developmente of biocatalytic processes. Organiz process research e development, v. 17, p. 1233-1238, 2013.

YALCIN, S. K.; BOZDEMIR, M. T.; OZBAS, Z. Y. A comparative study on citric acid production kinetics of two Yarrowia lipolytica strains in two different media. Indian Journal of Biotechnology, v. 8, p. 408-417, 2009.

WEHMER, C. Darstellung von citronensaure mittels Gärung. Chem Zentr, v. 2, p. 1252-1256, 1893. 